\title{
Theoretical Prediction of Lanthanum Composition Effects on Structural, Electronic and Thermal Properties of $\mathrm{La}_{\mathrm{x}} \mathrm{Sc}_{1-\mathrm{X}} \mathrm{N}$ Alloys.
}

Amira EL-HASSASNA ( $\sim$ amira.el-hassasna@univ-tebessa.dz )

Universite Larbi Tebessi: Universite de Tebessa https://orcid.org/0000-0003-4778-3113

CHAOUCHE Yassine

Universite Larbi Tebessi: Universite de Tebessa

Louafi Ahd

Universite Larbi Tebessi: Universite de Tebessa

\section{Research Article}

Keywords: Density functional theory, FP-LAPW, electronic band structure, thermal properties, Debye model.

Posted Date: July 14th, 2021

DOl: https://doi.org/10.21203/rs.3.rs-662795/v1

License: (c) (i) This work is licensed under a Creative Commons Attribution 4.0 International License. Read Full License 


\section{Abstract}

In this paper we have study the structural, electronic and thermal properties of $\mathrm{La}_{\mathrm{x}} \mathrm{Sc}_{1-\mathrm{x}} \mathrm{N}$ ternary alloys in rock-salt structure by the use of full potential linearized augmented plane wave (FP-LAPW) method based to the density functional theory (DFT). For calculate the exchange-correlation energy and potential we are used both the Wu-Cohen generalized gradient (WC-GGA) approximation and the modified BeckeJohnson(mBJ).We investigated the fact of composition on lattice parameters, bulk modulus and band gap. The variation of calculated lattice constant with lanthanum composition is almost linear and shows a small deviation of the obtained results from Vegard's law. The semiconductor nature of binary compounds $\mathrm{ScN}$ and $\mathrm{LaN}$ was confirmed. Our finding indicates that the $\mathrm{La}_{x} \mathrm{Sc}_{1-x} \mathrm{~N}$ alloys are semiconductor with $x=0.5$ and 0.75 , on the contrary for $x=0.25$ has a metallic nature. Lastly, the effect of thermal macroscopic properties is also investigated employing the quasi-harmonic Debye model in which the lattice vibrations are taken into account. We have found a good accord between our results and the experimental data and previous theoretical results available in the literature for the binary compounds which can be a support for the ternary alloys in the future.

\section{Declarations}

'Not applicable' for that section.

Funding (No funding was received to assist with the preparation of this manuscript.)

Conflicts of interest/Competing interests (There is no conflicts of interest)

\section{Introduction}

The studied of different physical properties through quantum mechanical methods such as the first principle calculation which it is the most used approach in the simulation methods that are based on the density functional theory (DFT). The most quantum mechanical approach used is the DFT functional and it can be applied to the majority of atoms and molecules called finite systems and surfaces and solids called infinite systems; This computer technique is a general purpose to determine different properties of solids as the structures of crystal and energy barriers to diverse methods with low computational rate and reasonable precision [1].

In recent years, attention has been paid to alloys and the study of their physical properties such as electronic, structural, elastic and thermodynamic properties. The thermophysical properties; the isothermal bulk modulus, coefficient of thermal expansion, heat capacity, Debye temperature and Grüneisen parameter has been attracted the attention of researchers for many years especially for alloys. Studying these properties enables us to develop these alloys to become more effective and useful in the industrial and electronic fields and many more [2-5]. 
The early transition metal (mono) nitrides have attracted the attention of many researchers which are a compounds crystallize in the rock-salt structure(B1) at normal temperature and pressure with the possibility of a transition to the $\mathrm{CsCl}$ (B2) at high pressures and can be a metallic or semiconducting have a large range of technological applications; mechanical strength, high hardness, high melting point, and relatively high superconducting transition temperatures, These properties make it enable to use them in technological applications like the area of hard coatings and magnetic storage device and cutting tools, due to their excellent physical properties[6-8].

The structural and electronic properties of ScN have been determined by Takeuchi et al. using firstprinciples calculations in the rock-salt, cesium chloride, nickel arsenide, zinc-blende, and wurtzite structures [9, 10]. In 2019 the structural and electronic properties of ScN, YN, LaN, and LuN has been studied by Maciej J Winiarski and DorotaKowalska [11] using the density functional theory and the band structures were calculated using the modified approach of Beck-Johnson, also Mohamed Ghezali et al. [12] investigated the structural and electronic properties of LaN compound using two different methods a version of the full potential linear Muffin-tin orbitals method (FP-LMTO) and the full potential linear augmented plane waves (FP-LAPW). Then A. Gueddim et al [13] studied the parameters of crystal structure and elastic coefficients of LaN compound at null and raise pressure using the density functional theory within both local density approximation (LDA) and generalized gradient approximation (GGA), and A. Louhadj et al [14] study the electronic band structures of ScN, YN, LaN and GdN compounds employing the full potential linear Muffin-tin orbital method (FPLMTO) within the local density approximation (LDA).

LaN compound is one of the most important materials which attracted researchers, it is a transition metal nitride and sometimes it can be a superconductor, it is crystallized in the rock salt (B1) phase and under pressure have a probable phase change to the $\mathrm{CsCl}$ [12]. The $\mathrm{ScN}$ compound has a rock salt structure (B1) with a possible transition to the $\mathrm{CsCl}$ (B2) phase as function of pressure and both possibly have a metastable Wurzite (B4) phase [15].

The aims of this current study, is to calculate the structural, electronic and thermal properties of $\mathrm{La}_{1-\mathrm{x}} \mathrm{Sc}_{\mathrm{x}} \mathrm{N}$ alloys for $x$ compositions ranging from $x=0,0.25,0.5,0.75$ and 1 . The contribution purposes to investigate the effect of lanthanum composition on structural, electronic and thermal properties of $\mathrm{La}_{1-x} \mathrm{Sc}_{x} \mathrm{~N}$ alloys. Therefore, the present contribution wishes to provide more information in the literature on the basic properties of $\mathrm{La}_{1-\mathrm{x}} \mathrm{Sc}_{\mathrm{x}} \mathrm{N}$, which can be serving as references for future experimental research on these systems. This allows the development of novel materials and improving the limitation of existing devices in electronic and optoelectronic technological applications. This study is accomplished by the Wu-Cohen generalized gradient approximations (WC-GGA) and modified Becke-Johnson (mBJ) included the full potential linearized augmented plane wave method (FP-LAPW) in the framework of the density functional theory (DFT).In section 2, the method of calculation is briefly outline depicted. The theoretical results obtained for the structural, electronic and thermal properties of $\mathrm{La}_{1-x} \mathrm{Sc}_{x} \mathrm{~N}$ ternary alloys and discussion are presented in section 3 , followed by a conclusion of this work in section 4 . 


\section{Method Of Calculation}

The calculations of total energy have been carried out using the scalar non-relativistic full-potential linearized augmented plane wave method (FP-LAPW) [16] as implemented in wien2k computational package [17], based on the density functional theory (DFT) $[18,19]$. The exchange-correlation potential is treated using the Wu-Cohen generalized gradient approach (WC-GGA) [20], the modified Becke and Johnson potential $(\mathrm{mBJ})$ has been used $[21,22]$ in order to calculate the electronic structure. We have applied the Special Quasi-random structures (SQS) technique proposed by Zunger et al. [23] to reproduce the randomness of the alloys for the initial some shells about a given site. The reasonable precise of (SQS) scheme to define the different properties, physical and chemical, which the introduced errors are not attained, it uses beyond the initial few shells by the model of periodicity. The electron wave function was expended inside the muffin-tin spheres (MT) in mixture with a cut-off $I_{\max }=10$ as spherical harmonic functions, also the charge density was Fourier expended up to $G_{\max }=12$ (Ryd) ${ }^{1 / 2} \cdot R_{M T} K_{\max }$ was determined with a value of 8 , where $R_{M T}$ is the plane wave radius and $K_{\max }$ is cut-off for wave function basis and we chose the energy between the core and valence states for cut-off about -6.0 Ry for. The values of muffin-tin radii $\left(R_{M T}\right)$ were chosen to be $2.55,2$, and $1.7(\mathrm{au})$ for the La, Sc and $\mathrm{N}$ atoms respectively. An irreducible Brillouin zone mesh of 1331 special $\mathrm{K}$ points have been used for the both kinds of materials, binary and ternary alloys. We are studied the thermal effects by employing the quasiharmonic Debye model [24] implemented in the code Gibbs2 program.

\section{Result And Discussion}

\subsection{Structural properties}

Firstly, we determine the structural properties of the binary compounds $\mathrm{LaN}, \mathrm{ScN}$ in rock-salt structure employing the WC-GGA approximation, and the ternary alloys $\mathrm{La}_{1-\mathrm{x}} \mathrm{Sc}_{x} \mathrm{~N}$ are modeled at different concentrations, as repeated supercells for the lanthanum concentrations $x=0.25,0.5$ and 0.75 in terms of periodically with the described ordered structures. Thus, the structure of ternary alloys is determined by super-cells of 8 -atoms corresponding to $1 \times 1 \times 1$ single cells. The structural optimization was realized by minimizing the total energy of cell parameters and the atomic positions. In order to determine the optimal lattice parameter (a), the bulk modulus (B) and its pressure derivative (B'), we calculated the total energies at various volumes in the region of equilibrium and by means of the Birch-Murnaghan equation of state [25] we fitted the values. The calculated structural parameters for $\mathrm{La}_{x} \mathrm{Sc}_{1-\mathrm{x}} \mathrm{N}$ at different compositions $\mathrm{x}=0,0.25,0.5,0.75$ and 1 employing WC-GGA approach are presented in table 1, together with available experimental measurement and theoretical results reported in the literature for comparison. Our computed structural parameters for binary compounds $\mathrm{ScN}$ and $\mathrm{LaN}$, are similar with very little variation to the theoretical works reported previously. In comparison to the experimental result, the deviation of our results from lattice constants is less than $0.7 \%$ and $0.8 \%$ within $\mathrm{ScN}$ and LaN respectively, there are weakly overestimated with respect to the experiment. This result confirms the usual trend of GGA approximation to overestimate lattice constant value [26-28]. Whereas, the bulk modulus value of ScN is 
in excellent accord between our results and that of 215.86GPacalculated by Shoaib et al in [7] using the density functional theory based FP-LAPW method, while forLaN, our results are larger than $13 \%$ with experimental value of $112 \mathrm{GPa}$ presented in [29]. On the other hand, we note that the larger lattice parameter for $\operatorname{LaN}(x=1)$ than those of $\operatorname{ScN}(x=0)$ compounds where $a(\operatorname{LaN})>a(\operatorname{ScN})$, while the same anion atom in both compounds. These results can be clarified by taking in consideration the atomic radii of the $\mathrm{La}$ and $\mathrm{Sc}$ atoms, which are given by $\left(R(L a)=2.74 \mathrm{~A}^{\circ}\right)$ and $\left(R(S c)=2.09 \mathrm{~A}^{\circ}\right)$ respectively.

The bulk modulus value for $\mathrm{ScN}$ is larger than those of $\mathrm{LaN}, B(\mathrm{ScN})>B(\mathrm{LaN})$; while the inverse decreases of the bulk modulus value to the lattice parameter $a$, in agreement the well-known liaison between $B$ and the lattice constant $B a V_{0}^{-1}$ where $V_{0}$ is the volume of unit cell [30]. Because of the absence of experimental and theoretical works concerning lattice parameters and bulk modulus for alloys composition $x(0 \otimes x \otimes 1)$, we consider that our results for this composition are predicted work and may be considered as references for future.

The studied of variation lattice parameter for $\mathrm{La}_{\mathrm{x}} \mathrm{Sc}_{1-\mathrm{x}} \mathrm{N}$ alloy with La concentration $\mathrm{x}$ is seen in figure 1 . This variation is approximately linear and shows an important deviation from Vegard's law [31] with downward bowing parameter equal to $-0.297 \mathrm{~A}^{0}$; this behavior is due to the mismatches of lattice constant of ScN and LaN compounds. In figure 2, we display the results of the bulk modulus at different concentration $x$ for $\mathrm{La}_{x} \mathrm{Sc}_{1-\mathrm{x}} \mathrm{N}$, Between the different values of bulk modulus of the extreme binary compounds ScN and LaN, we remark a deviation of the bulk modulus with downward bowing equal to $96.691 \mathrm{GPa}$, this difference is generally caused by the mismatch of the bulk modulus of binary compound. in addition, the decreasing of bulk modulus with increasing La composition from 0 to 1 indicating that the $\mathrm{La}_{\mathrm{x}} \mathrm{Sc}_{1-\mathrm{x}} \mathrm{N}$ alloys becomes more compressible as the concentrations $\mathrm{x}$ increases.

Table 1: Computed lattice constants $a_{0}\left(\mathrm{~A}^{\circ}\right)$, bulk modulus $B(\mathrm{GPa})$ and its pressure derivative of $\mathrm{La}_{\mathrm{x}} \mathrm{Sc}_{1}$ ${ }_{x} \mathrm{~N}$. 


\begin{tabular}{|c|c|c|c|c|c|c|c|c|}
\hline \multirow[t]{2}{*}{$x$} & \multicolumn{3}{|l|}{$a_{0}\left(\mathrm{~A}^{\circ}\right)$} & \multicolumn{3}{|l|}{$B(\mathrm{GPa})$} & \multicolumn{2}{|l|}{$B^{\prime}$} \\
\hline & Thiscal. & Other cal. & Exp & $\begin{array}{l}\text { This } \\
\text { Cal. }\end{array}$ & Other cal. & Exp & $\begin{array}{l}\text { This } \\
\text { cal. }\end{array}$ & $\begin{array}{l}\text { Other } \\
\text { cal. }\end{array}$ \\
\hline \multirow[t]{4}{*}{0} & 4.471 & & & 213.001 & $201^{d}$ & & 4.158 & $3.31^{d}$ \\
\hline & & $4.516^{c}$ & $4.44^{\mathrm{a}}$ & & $215.86^{e}, 235^{f}$ & & & $3.65^{\mathrm{e}}$ \\
\hline & & 4.04 & $4.51^{\mathrm{b}}$ & & & & & \\
\hline & & $4.48^{e}, 4.50^{f}$ & & & & & & \\
\hline 0.250 & 4.728 & - & & 168.444 & - & & 3.926 & - \\
\hline 0.500 & 4.938 & - & & 148.446 & - & & 3.897 & - \\
\hline 0.750 & 5.060 & - & & 136.252 & - & & 3.793 & - \\
\hline \multirow[t]{3}{*}{1} & 5.257 & $5.26^{e}$ & & 130.11 & $130.92^{\mathrm{e}}$ & & 4.124 & $3.84^{\mathrm{e}}$ \\
\hline & & $5.32^{f}$ & $5.3^{9}$ & & $148^{f}$ & $112^{\mathrm{h}}$ & & $4.01^{i}$ \\
\hline & & $5.28^{i}$ & & & $121.1^{i}$ & & & \\
\hline
\end{tabular}

$\operatorname{Ref}^{\mathrm{a}}$ [32], $\operatorname{Ref}^{\mathrm{b}}[33], \operatorname{Ref}^{\mathrm{c}}[34], \operatorname{Ref}^{\mathrm{d}}[9], \operatorname{Ref}^{\mathrm{e}}[7], \operatorname{Ref}^{f}[6], \operatorname{Ref}^{\mathrm{g}}[35], \operatorname{Ref}^{\mathrm{h}}[29], \operatorname{Ref}^{\mathrm{i}}[36]$.

\subsection{Electronic properties}

We have computed the electronic band structures of $\mathrm{ScN}$ and LaN binary compounds and their ternary alloys $\mathrm{La}_{\mathrm{x}} \mathrm{Sc}_{1-\mathrm{x}} \mathrm{N}$ in rock-salt structure for the concentration $\mathrm{x}=0.25,0.5$ and 0.75 using the optimized lattice constant value along the high symmetry directions in the first Brillouin zone within both WC-GGA and $\mathrm{mBJ}$ approaches.

The band structure of ScN and LaN compounds obtained by $\mathrm{mBJ}$ approximation are displayed in figure 3. In figure 3(a) we are seen the indirect band gap of $\mathrm{ScN}, \mathrm{E}_{\mathrm{g}}(\Gamma-\mathrm{X})=0.918(\mathrm{eV})$, our results is in accord with the previous LDA(mBJ) 0.9-1.0 (eV) [37,38] and 1.0(eV) [39] works. Interestingly, the optical spectroscopy measurements for $\mathrm{ScN}$ suggested a higher experimental band gap of 1.3(eV) [40]. On the other hand, the electronic band structure of $\mathrm{LaN}$ is also represented in figure 3(b). We remark the highest of the valence band (VB) is located at $X$ high symmetry point and the smallest of the conduction band (CB) is situated at X point in Brillouin zone. This is coherent with the fact that $L a N$ is a direct $(X-X)$ band gap semiconductor with a value of $0.694(\mathrm{eV})$, which is close to the experimental value of band gap $E_{g(e x p)}=0.75(e V)[6]$ and is in good accord with the theoretical value of 0.73(eV) [7] and $0.59(\mathrm{eV})$ [11] results. Otherwise, the binary compounds are a metallic capture using WC-GGA approximation. We can note, in the calculated values of the electronic band gap, that the variance compared to the experimental value is explained probably by the fact of GGA potential of exchange and correlation included in the 
density functional theory which it underestimates the value of energy gap in semiconductors, due to the self-interaction error and the absence of derivative discontinuity in the exchange and correlation potential, for this raison the energy band gap can be underestimated up to 50\% [41-43]. The results for electronic band structure obtained for ternary alloys $\mathrm{La}_{x} \mathrm{Sc}_{1-\mathrm{x}}$ Nwith La concentrations $\mathrm{x}(0<\mathrm{x}<1)$ within TB-mBJ approximation are displayed in figure 4 .We can be seen the direct band gap of $\mathrm{La}_{0.5} \mathrm{Sc}_{0.5} \mathrm{~N}$ and $\mathrm{La}_{0.75} \mathrm{Sc}_{0.25} \mathrm{~N}$ at $\Gamma$ point equal to0.02 (eV) and $0.38(\mathrm{eV})$ values respectively, while the $\mathrm{La}_{0.25} \mathrm{Sc}_{0.75} \mathrm{Nalloy}$ indicates the metallic character with a negative gap of $-0.004(\mathrm{eV})$,the calculate band structure using WCGGA approximation indicate the metallic character for La compositions $x(0<x<1) \mathrm{inLa}_{x} \mathrm{Sc}_{1-x} \mathrm{~N}$. we have presented our calculated band gap energies for $\mathrm{La}_{\mathrm{x}} \mathrm{Sc}_{1-\mathrm{x}} \mathrm{N}$ ternary alloys at different concentrations using $\mathrm{mBJ}$ approach in teble2 compared to experimental and previous published theoretical results. For La concentrations $x=0.25,0.5$ and 0.75 in $\mathrm{La}_{\mathrm{x}} \mathrm{Sc}_{1-\mathrm{x}} \mathrm{N}$ ternary alloys, our results are predictions.

Table2: The calculated band gapenergy (in $\mathrm{eV}$ ) at high symmetry points for $\mathrm{La}_{\mathrm{x}} \mathrm{Sc}_{1-\mathrm{x}} \mathrm{N}$ ternary alloys at different La concentrations $x$ computed using $\mathrm{mBJ}$.

\begin{tabular}{|c|c|c|c|}
\hline \multicolumn{4}{|c|}{$E_{g}(e V)$} \\
\hline$x$ & Our Cal & Exp & Other \\
\hline 0 & $0.918(\Gamma-X)$ & $1.3^{\mathrm{a}}$ & $0.9^{b}, 1.0^{c}, 1.0^{d}$ \\
\hline 0.25 & $-0.004(Г-Г)$ & - & - \\
\hline 0.5 & $0.02(Г-Г)$ & - & - \\
\hline 0.75 & $0.38(Г-\Gamma)$ & - & - \\
\hline 1 & $0.694(X-X)$ & $0.75^{\mathrm{e}}$ & $0.73^{\mathrm{f}}, 0.59^{\mathrm{g}}$ \\
\hline
\end{tabular}

$\operatorname{Ref}^{a}[40], \operatorname{Ref}^{b}[37], \operatorname{Ref}^{c}[38], \operatorname{Ref}^{d}[39], \operatorname{Ref}^{\mathrm{e}}[6], \operatorname{Ref}^{f}[7], \operatorname{Ref}^{\mathrm{g}}[11]$.

In arrange to clarify the character of the electronic band structures of $\mathrm{La}_{\mathrm{x}} \mathrm{Sc}_{1-\mathrm{x}} \mathrm{N}$ ternary alloys, we have computed the partial density of states at different La concentrations using $\mathrm{mBJ}$ approaches, our results are presented in figure 5 . We can be remarked three separate regions for the partial density of states of $\mathrm{La}_{\mathrm{x}} \mathrm{Sc}_{1-\mathrm{x}} \mathrm{Nf}$ or the concentration $\mathrm{x}=0.25,0.5$ and 0.75 . The two lowest regions constitute the energy distribution of the valence band electrons below Fermi level $\left(E_{F}\right)$ and the highest region above $E_{F}$ is the conduction band.

For $\mathrm{La}_{0.25} \mathrm{Sc}_{0.75} \mathrm{~N}$ the lowest valence band in the energy range around $-16.5 \mathrm{eV}$ up to $14.5 \mathrm{eV}$ and around $-14 \mathrm{eV}$ to $10.5 \mathrm{eV}$, are due to Sc-p states, while the s states of $\mathrm{N}$ are localized around $-14.5 \mathrm{eV}$ to $-14 \mathrm{eV}$, the highest valence band near the Fermi level around $-5 \mathrm{eV}$ to $0 \mathrm{eV}$ is mainly derived from N/La-p states and weak contribution of La-d states and Sc-p/d states. The conduction band is formed by Sc-d states 
and a small contribution of La-p/d states and $\mathrm{N}-\mathrm{s} / \mathrm{p}$ states. We observe that the metallic character of the $\mathrm{La}_{0.25} \mathrm{Sc}_{0.75} \mathrm{~N}$ alloy is principally determined by the $\mathrm{d}$ states of La atoms and $\mathrm{p}$, $\mathrm{d}$ states of $\mathrm{Sc}$ atoms.

For $\mathrm{La}_{0.5} \mathrm{Sc}_{0.5} \mathrm{~N}$, the lowest valence band extending between $-16.5 \mathrm{eV}$ to $-14.5 \mathrm{eV}$ and $-14 \mathrm{eV}$ to $-10.5 \mathrm{eV}$ is due to the $\mathrm{La} / \mathrm{Sc} / \mathrm{N}$-s states, around $-14 \mathrm{eV}$ to $-14 \mathrm{eV}$ is derived by a little Sc-s/p/d states, the highest valence band extends from $-5 \mathrm{eV}$ to $0 \mathrm{eV}$ is mainly formed by La/Sc/N-p states and a contribution of $\mathrm{La} / \mathrm{Sc}-\mathrm{d}$ states. The conduction band is formed by mixed La/Sc-p states and N-s states.

For $\mathrm{La}_{0.75} \mathrm{Sc}_{0.25} \mathrm{~N}$ the contribution of $\mathrm{La} / \mathrm{Sc} / \mathrm{N}$-s states is observed in the lowest valence band around $-16.5 \mathrm{eV}$ to $-14 \mathrm{eV}$ and between $-13 \mathrm{eV}$ to $-11 \mathrm{ev}$,but the highest valence band near the Fermi level around -4 $\mathrm{eV}$ to $\mathrm{Oev}$ is derived from $\mathrm{La} / \mathrm{Sc} / \mathrm{N}-\mathrm{p}$ states and a weak contribution of $\mathrm{La} / \mathrm{Sc}-\mathrm{d}$ states. The conduction band is characterized by mixed La/Sc-p and d states and N-p states.

\subsection{Thermal properties}

Is a necessary step to study the thermal properties of materials in order to determine their specific behavior, when they are submitted to constraints such as high temperature and high pressure. In this section, we use the quasi-harmonic Debye model of crystals [24] implemented in the Gibbs2 program and combined with the full potential linearized augmented plane wave FP-LAPW method, to determine the thermal properties of $\mathrm{La}_{x} \mathrm{Sc}_{1-\mathrm{x}} \mathrm{N}$ alloys. The energy volume data are calculated from the Britch-Murnaghan equation of states using WC-GGA approximation, these data are the inputs data to Gibbs2 program to calculate the thermal properties of the $\mathrm{La}_{\mathrm{x}} \mathrm{Sc}_{1-\mathrm{x}} \mathrm{N}$ alloys as function of temperature, in the range of $\mathrm{OK}$ to $2000 \mathrm{~K}$. This present study of the thermal properties is the first theoretical prediction in our case of studied alloys.

In figures 6 and 7 we present the variation of the volume and bulk modulus of $\mathrm{La}_{x} \mathrm{Sc}_{1-x} \mathrm{~N}$ alloys at $0 \mathrm{GPa}$ respectively. We notice that the effect of the temperature $T$ on the volume at every concentration is almost stable with the raise of temperature. However the bulk modulus varies linearly in a very moderate way for La concentrations at $x(0<x<1)$ in $\mathrm{La}_{x} \mathrm{Sc}_{1-\mathrm{x}} \mathrm{N}$ but the binary compounds is nearly constant from 0 to $200 \mathrm{~K}$, and diminishes with raising temperature at $\mathrm{T}>200 \mathrm{~K}$. Therefore the binary compounds $\mathrm{ScN}$ and LaN becomes more compressible with increasing temperature.

Figure 8 present the effect of temperature $T$ on the Debye temperature $\theta_{D}$ for the $\mathrm{La}_{\mathrm{x}} \mathrm{Sc}_{1-\mathrm{x}} \mathrm{N}$ alloys, it can be seen clearly from this figure that the value of Debye temperature is almost constant for $x=0.25,0.5$ and 0.75 , but for binary compounds at $\mathrm{T}>200 \mathrm{~K} \theta_{D}$ decrease linearly with increasing temperature. From figure 9 we can see that for a given temperature, $\theta_{D}$ increases linearly with increasing pressure. Our results present that $\mathrm{ScN}$ has a higher Debye temperature than the LaN, and predict that bulk modulus is higher for ScN than for LaN, suggesting that $\mathrm{ScN}$ is harder than LaN.

The computed heat capacity $\left(\mathrm{C}_{\mathrm{V}}\right)$ for the $\mathrm{La}_{\mathrm{x}} \mathrm{Sc}_{1-\mathrm{x}} \mathrm{N}$ alloys at $0 \mathrm{GPa}$ is presented in figure 10 , the evolution of the $C_{V}$ of $\mathrm{La}_{x} \mathrm{Sc}_{1-\mathrm{x}} \mathrm{N}$ at various compositions $\mathrm{x}=0,0.25,0.5$ and 0.75 is similar, also at $\mathrm{T}=0 \mathrm{~K}$ the heat 
capacity tends to zero; and increases with $\mathrm{T}^{3}$ at $\mathrm{T}<1600$ whereas the LaN compound increases with $\mathrm{T}^{3}$ at $T<1400$. The heat capacity $\left(C_{V}\right)$ results are depending on the temperature, due to the employing harmonic approximation within the Debye model which is used in our calculation. Otherwise, at superior temperature the harmonic effect on heat capacity vanishes, and also tends in constant value called Dulong-petit limit, which is similar to different materials at higher temperatures. Figure 11 determine the results for the $\mathrm{C}_{\mathrm{V}}$ as a function of pressure, indicating that, at different concentration, the values of higher temperature decrease approximately with increasing applied pressure.

The evolution of the heat capacity $\mathrm{C}_{\mathrm{p}}$ of $\mathrm{La}_{\mathrm{x}} \mathrm{Sc}_{1-\mathrm{x}} \mathrm{N}$ alloys at different concentrations with temperature and pressure is presented in figures 12 and 13 respectively. From figure 12 we observe the evolution of $C_{p}$ at low temperature is similar to that heat capacity $C_{V}$. However, at high temperature, the heat capacity $C_{p}$ exhibits a different characteristic, and the value of heat capacity does not converge to a constant values. It can be remark from figure 13 that for given temperature, the heat capacity $C_{p}$ decreases approximately linearly with increasing pressure.

The variation of entropy $(S)$ versus temperature is shown in figure 14; we can observe that the $S$ increases when the temperature increases for all compositions. This increases due to the fact that the increase in temperature increases the vibrational contribution to the entropy.

\section{Conclusion}

In this paper, we have studied the structural, electronics and thermal properties of $\mathrm{La}_{\mathrm{x}} \mathrm{Sc}_{1-\mathrm{x}} \mathrm{N}$ ternary alloys employing the FP-LAPW method farmed within WC-GGA and mBJ approximations. The calculated equilibrium lattice parameters and bulk modulus for the ScN and LaN were found to be in good agreement with the published theoretical and experimental results. The variation of the lattice parameter with La concentration was to have a small deviation from Vegard's law. The bulk modulus increases with alloy composition $\mathrm{x}$. the use of the $\mathrm{mBJ}$ approximation improved considerably the electronic band gap energy in comparison with experimental results. We have confirmed that both $\mathrm{ScN}$ and LaN, are semiconductors with an indirect gap $(\Gamma-\mathrm{X})$ and a direct gap $(X-X)$ respectively. The band gap is found to be direct $(\Gamma-\Gamma)$ at the concentration range from 0.5 and 0.75 but the alloy composition $x=0.25$ is metallic with a negative gap. The character of the electronic band structure for different concentrations $x$ was discussed from the stand point of the partial density of states. At last, the quasi-harmonic Debye model was successfully used to determine the thermal properties at different temperature in the range from 0 to $2000 \mathrm{~K}$ and pressures from 0 to $20 \mathrm{GPa}$. Our results presented in this manuscript are predictions and can be serving as references for future experimental study on these systems.

\section{References}

1. F.Tran, R., Laskowski, P., Blaha, K.: Schwarz,Phys.Rev.B 75, 115131 (2007) 
2. Suthar, R.K., Pandya, N.Y., Adwait, D., Mevada, P.N.: Gajjar.J. NANO AND E LECTRONIC P HYSICS.13, 01013 (2021)

3. ZheyuXie, B.W., Huang, J., Lin, J., Yang, Y., Jiang, L., Huang, J., Ye, G., Zhao, C., Yang, S., Sa, B.: Intermetallics. 93, 40 (2018)

4. Sklyarchuk, V., Plevachuk, Y., Yakymovych, A., Eckert, S., Gerbeth, G., Eigenfeld, K.: Thermophys. 30, 1400 (2009)

5. Kim, H., Boysen, D.A., Bradwell, D.J., Chung, B., Jiang, K., Tomaszowska, A.A., Wang, K., Wei, W.: and D. R. Sadoway. Electrochimica. Acta. 60, 154 (2012)

6. Stampfl, C., Mannstadt, W., Asahi, R.: A.J. Phys. Rev. B 63, 155106 (2001)

7. Shoaib, M., Murtaza, G., Khenata, R., Farooq, M., Ali, R.: Comput. Mater.Sci 79, 239246 (2013)

8. Tebboune, A.G., Rached, D., Benzair, A.N., Sekkal, N., Belbachir, A.H.: Phys. Status Solidi (b) 243,2788(2006)

9. Takeuchi, N., Ulloa, S.E.: Phys. Rev. B 65, 235307 (2002)

10. Takeuchi, N.: Phys. Rev. B 65, 045204 (2002)

11. Winiarski, M.J., Kowalska, D.: Mater. Res. Express 6, 095910 (2019)

12. Ghezali, M., Amrani, B., Cherchab, Y., Sekkal, N.: Mater. Chem. Phys. 112, 774 (2008)

13. Gueddim, A., Fakroun, N., Bouarissa, N., Villesuzanne, A.: Materials Chemistry and Physics. 118, 427 (2009)

14. Louhadj, A., Ghezali, M., Badi, F., Mehnane, N., Cherchab, Y., Amrani, B.: H. Abid and N.Sek kal. Superlatticesand Microstructures 46, 435 (2009)

15. Adachi, S.: GaAs and related materials. World Scientific Publishing Co. Pvt. Ltd (1999)

16. Anderson, O.K.: Phys. Rev. B42, 3060 (1975)

17. Blaha, P., Schwarz, K., Madsen, G.K.H., Kvasnicka, D., Luitz, J.: WIEN2K, An Augmented Plane Wave. Plus Local Orbitals Program For Calculating Crystal, Properties (Vienna, Austria (2008)

18. Hohenberg, P., Kohn, W.: Phys. Rev. B 136, 864 (1964)

19. Kohn, W., Sham, L.S.: Phys. Rev. A 140, 1133 (1965)

20. Wu, Z., Cohen, R.E.: Phys. Rev. B 73, 235116 (2006)

21. Tran, F., Blaha, P.: Phys. Rev. Lett. 102, 226401 (2009)

22. Becke, A.D., Johnson, E.R.: J. Chem. Phys. 124, 221101 (2006)

23. A.Zunger,S.-H..Wei,L.G.Feireira,J.E.Bernard: Phys.Rev.Lett 65, 353 (1990)

24. Blanco, M.A., Francisco, E., Luana, G.I.B.B.S.: Comput. Phys.Commun. 158, 57 (2004)

25. Murnaghan, F.D.: Proc. Natl. Acad. Sci. USA 30, 5390(1944)

26. Saib, S., Bouarissa: Phys. Status Solidi B 244, 1063 (2007)

27. Saib, S., Bouarissa: Solid State Electron. 50, 763 (2006)

28. Zerroug, S., Ali Sahraoui, F., Bouarissa: Eur. Phys. J. B 57, 9 (2007) 
29. Leger, J.M., Ravot, D., Rossat-Mignod, J.: J. Phys. C, Solid State Phys. 17, 4935 (1984)

30. Cohen, M.L.: Phys. Rev. B 32, 7988 (1985)

31. Vegard, L.: Z. Phys. 5, 17 (1921)

32. Wyckoff, R.W.G.: Cryst. Struct 1, 85 (1963)

33. Shirotani, I., Yamanashi, K., Hayashi, J., Ishimatsu, N., Shimomura, O., Kikegawa, T.: Solid State Commun. 127, 573 (2003)

34. Brik, M.G.: C.-G.Ma,Computational Materials Science 51, 380 (2012)

35. Amrani, B., Hassan, F.E.H.: Comput.Mater.Sci. 39, 563 (2007)

36. Ghezali, M., Amrani, B., Chercheb, Y., Sekkal, N.: Mater. Chem. Phys. 112, 774 (2008)

37. Zhang, S., Holec, D., FuWY, Humphreys, C.J., Moram, M.A.: Appl. Phys. 114, 133510 (2013)

38. Tran.F and Blaha, P.: Phys.Rev. Lett. 102, 226401 (2009)

39. Qteish, A., Rinke, P., SchefflerMand, Neugebauer: Phys. Rev. B 74, 245208 (2006)

40. Gall, D., Stadele, M., Jarrendahl, K., Petrov, I., Desjardins, P., Haasch, R.T., Lee, T.-Y., Greene, J.E.: Phys.

Rev. B 63, 125119 (2001)

41. Noor, N.A.: N.Ikram,S.Ali,S.Nazir,S.M.Alay-e-Abbas. A.Shaukat,Alloy.Compd. 507, 356 (2010)

42. Godby, R.W.: ,M.Schlüter,L.J.Sham. Phys.Rev.Lett 56, 2415 (1986)

43. Städle, M., Majevski, J.A.P.Vogel,A.Görling,Phys.Rev.Lett.79, 2089 (1997)

\section{Figures}

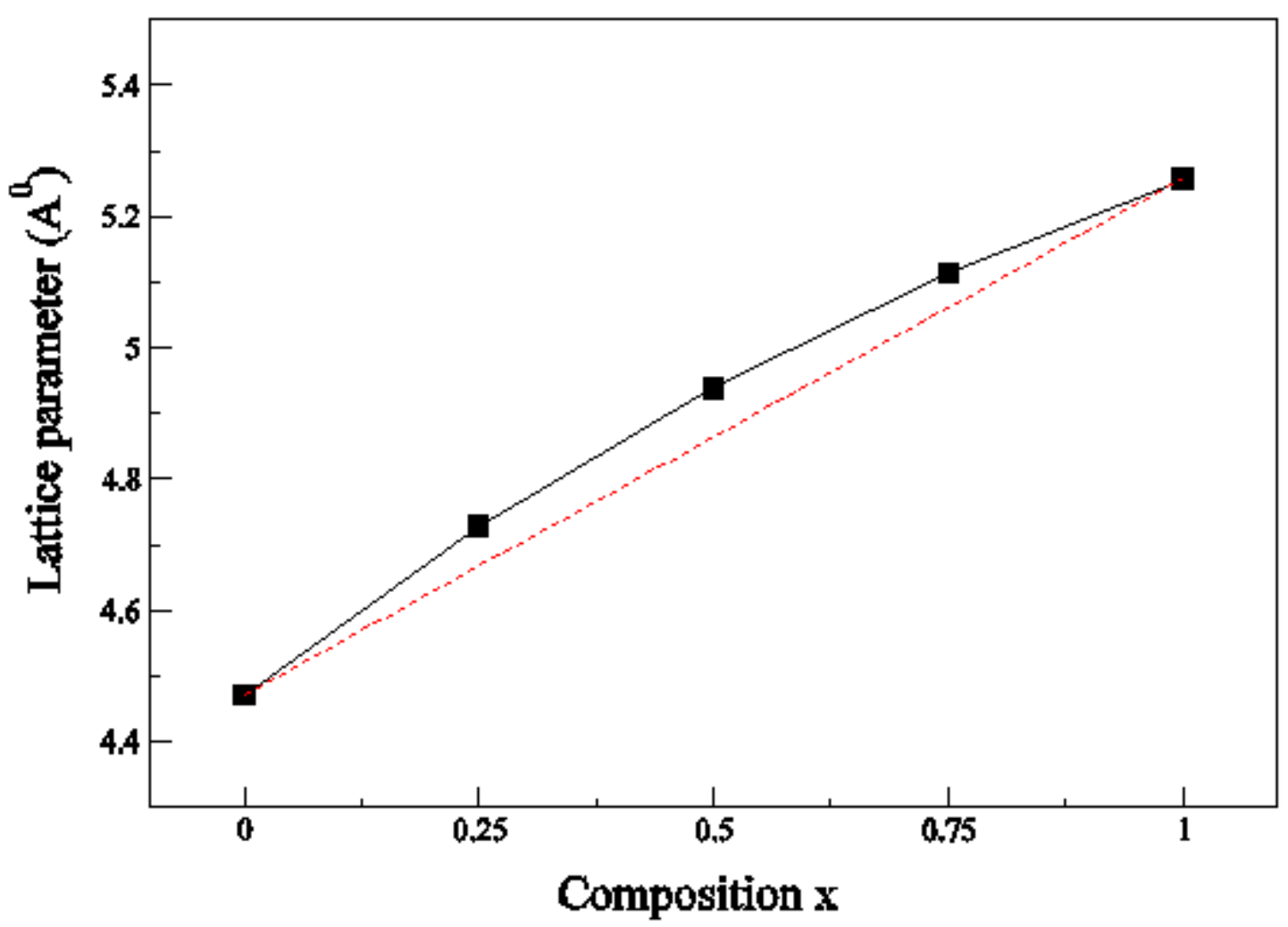

Page $11 / 20$ 
Figure 1

Lattice parameter as a function of La concentration (filled squares) compared with Vegard's law (dashed line).

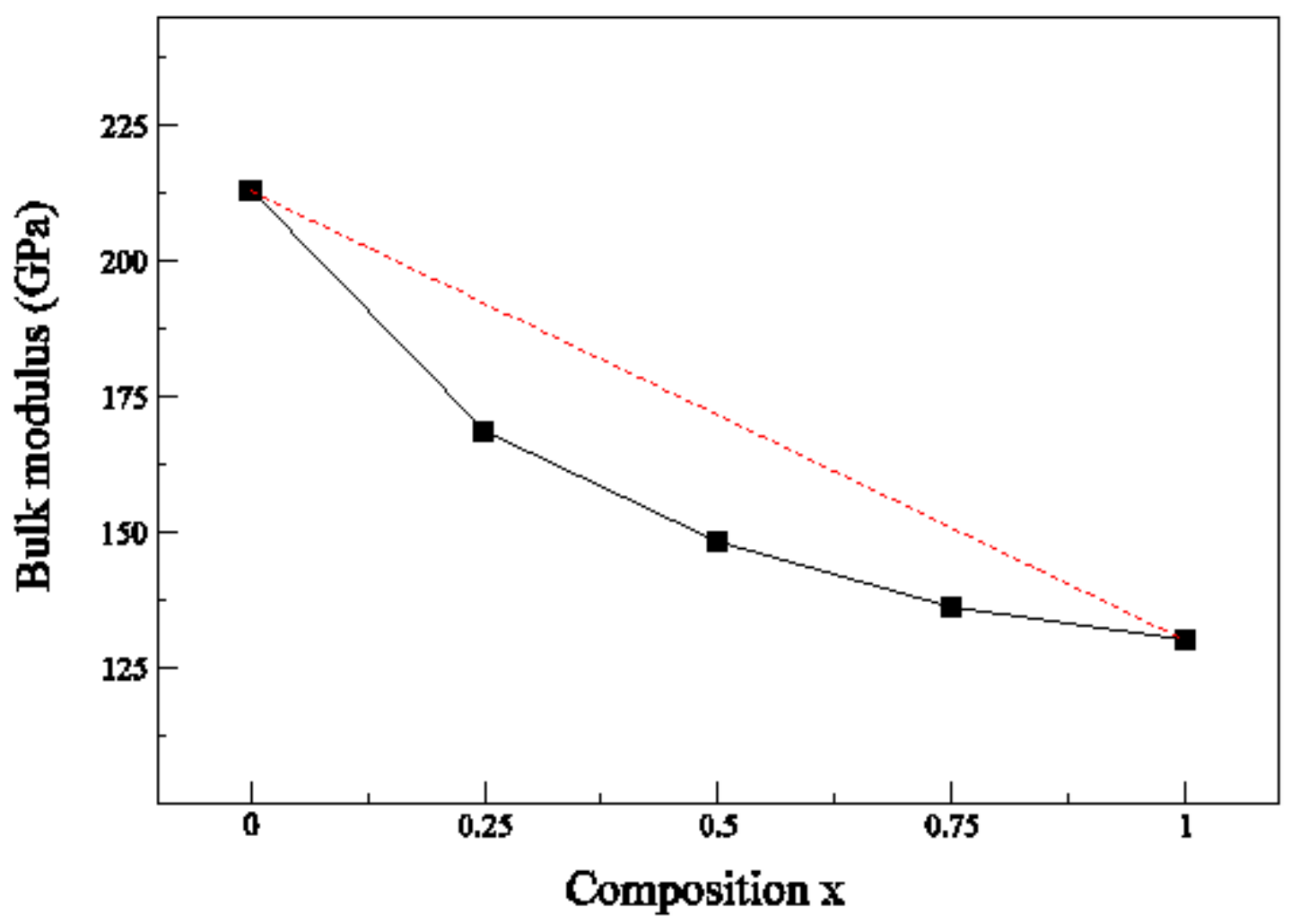

Figure 2

Bulk modulus as a function of La concentration (filled squares) compared with the linear composition dependence prediction (dashed line). 

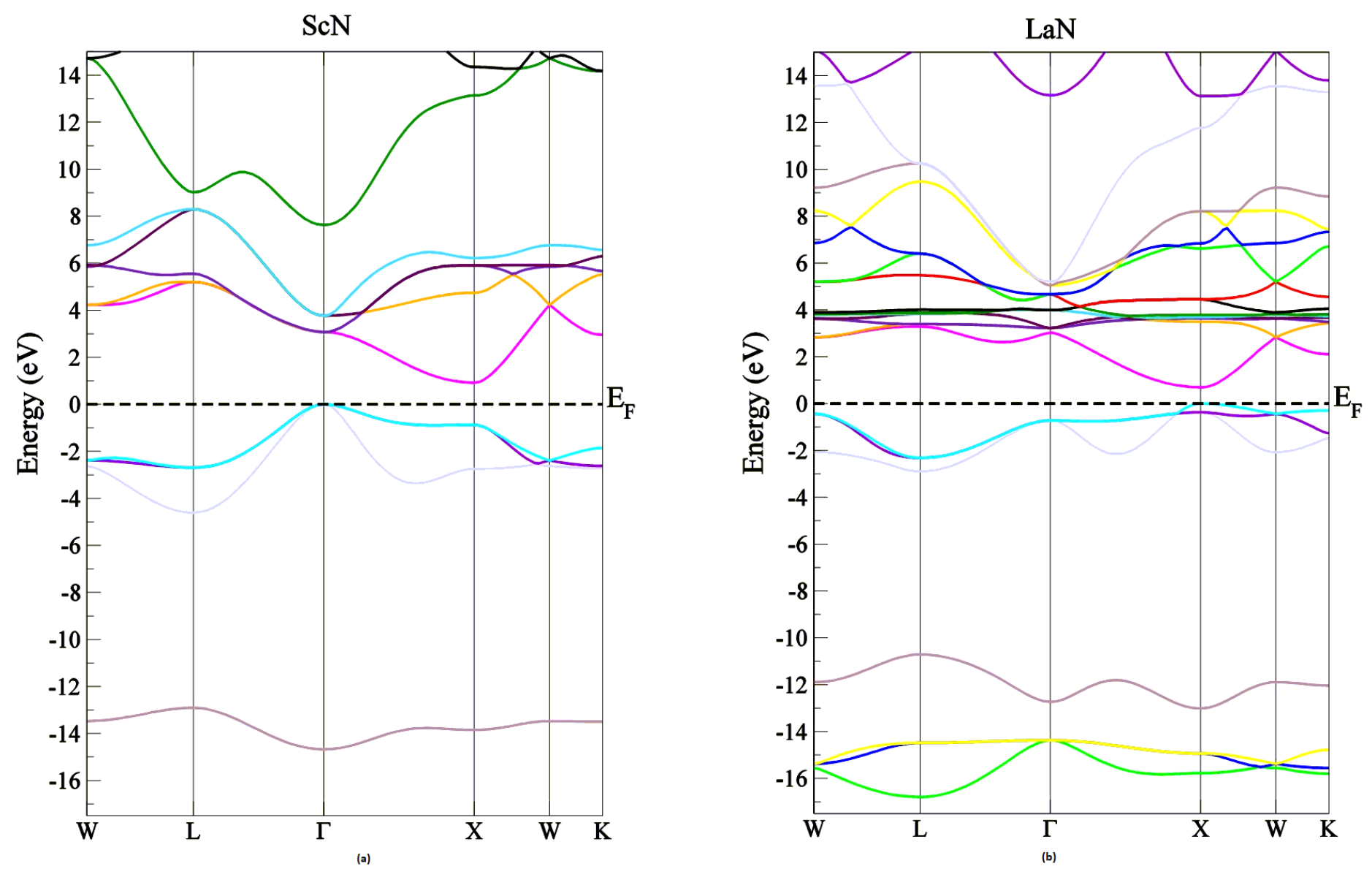

Figure 3

Band structure of ScN (a) and LaN (b) calculated within mBJ approximation. 

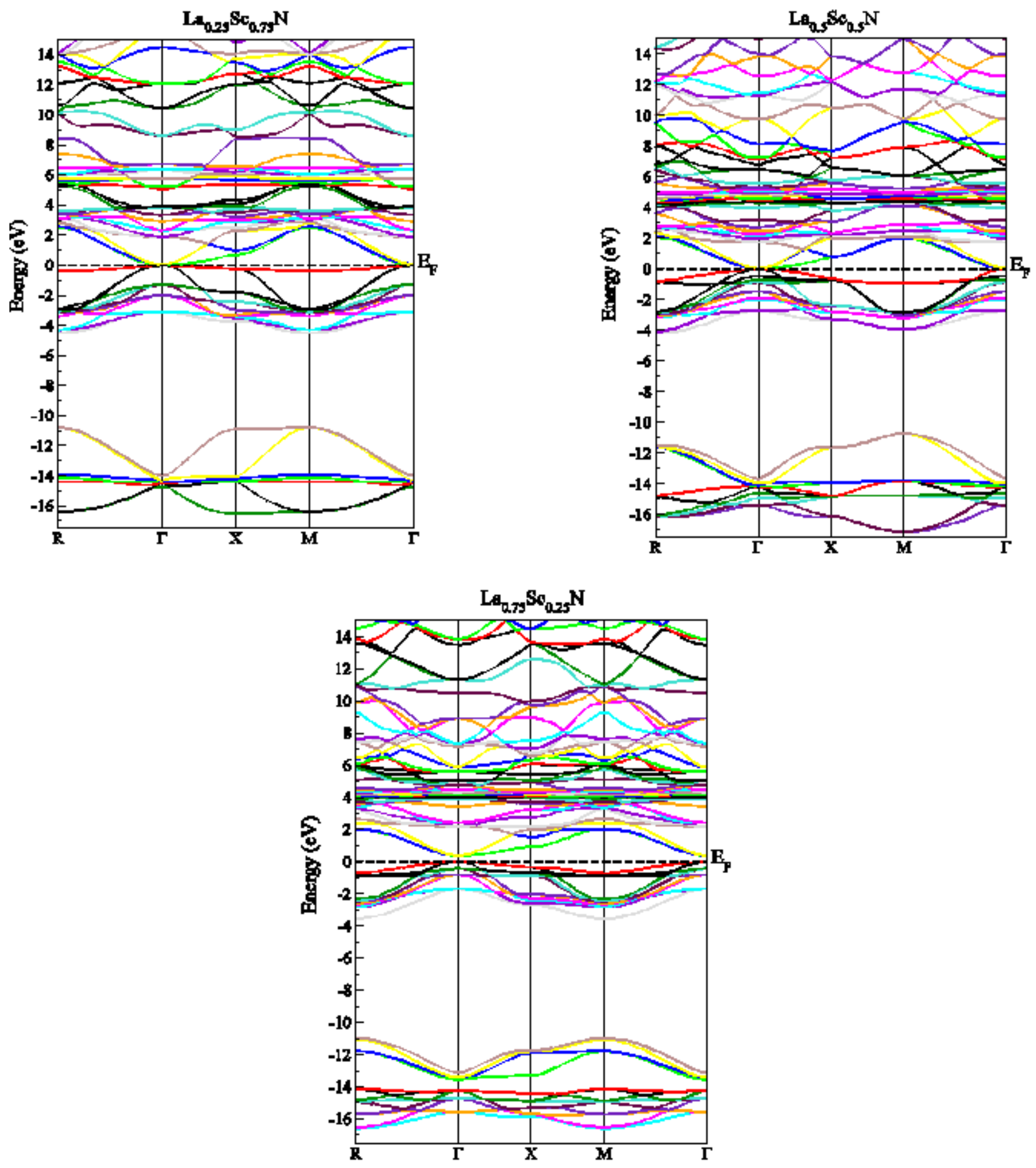

Figure 4

Band structure of LaxSc1-xN ternary alloys (at $x=0.25,0.5$ and 0.75 ) calculated within $\mathrm{mBJ}$ approximation. 

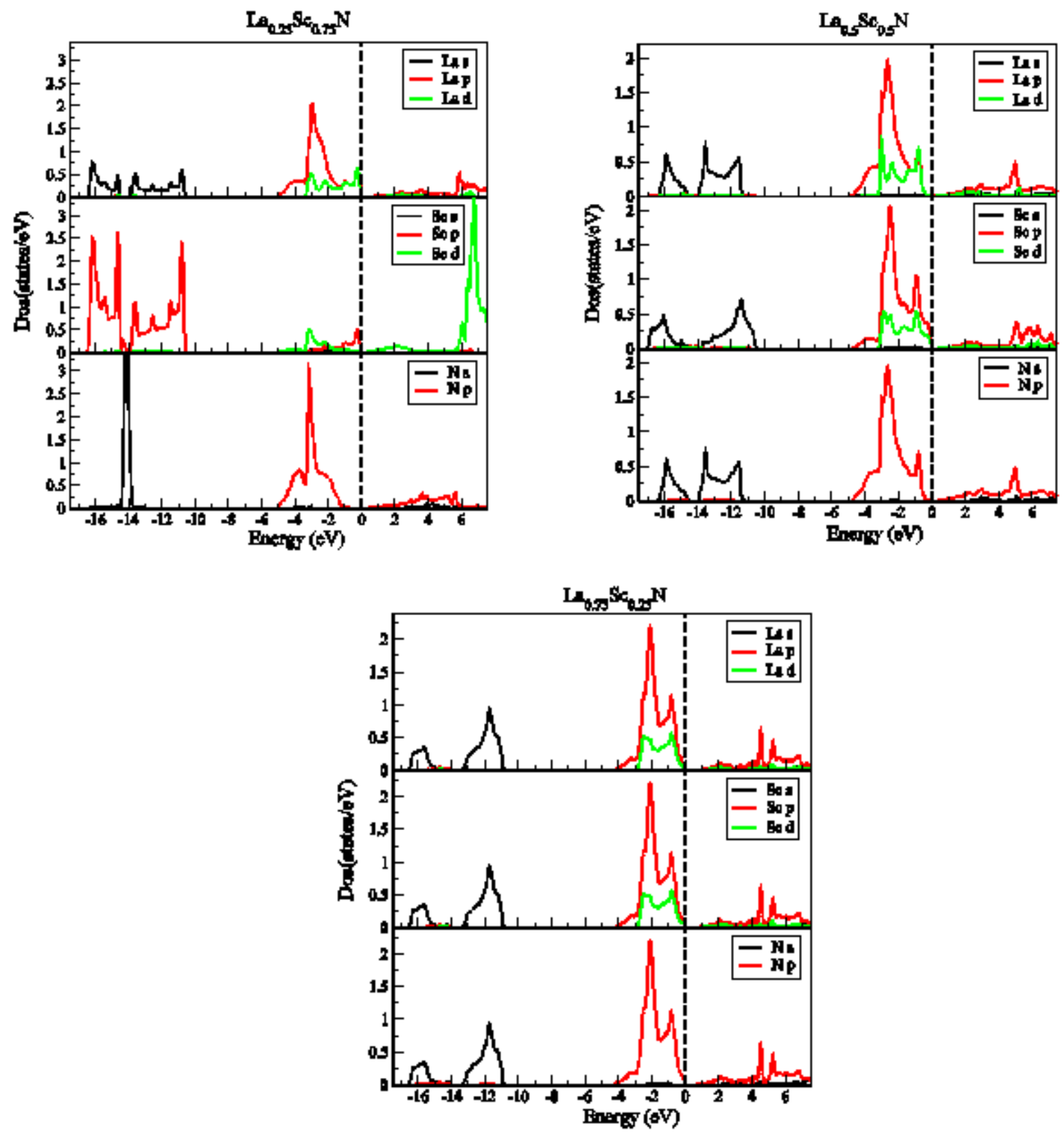

Figure 5

Partial density of states of LaxSc1-xN ternary alloys (at $x=0.25,0.5$ and 0.75 ) calculated within mBJ approximation. 


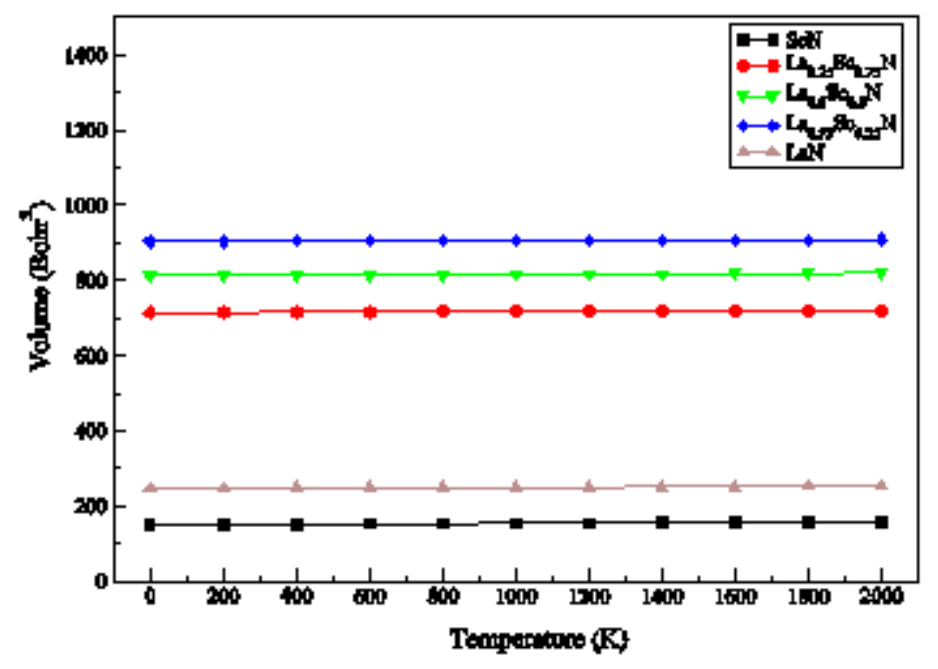

Figure 6

Variation with temperature of the volume for LaxSc1-xN alloys.

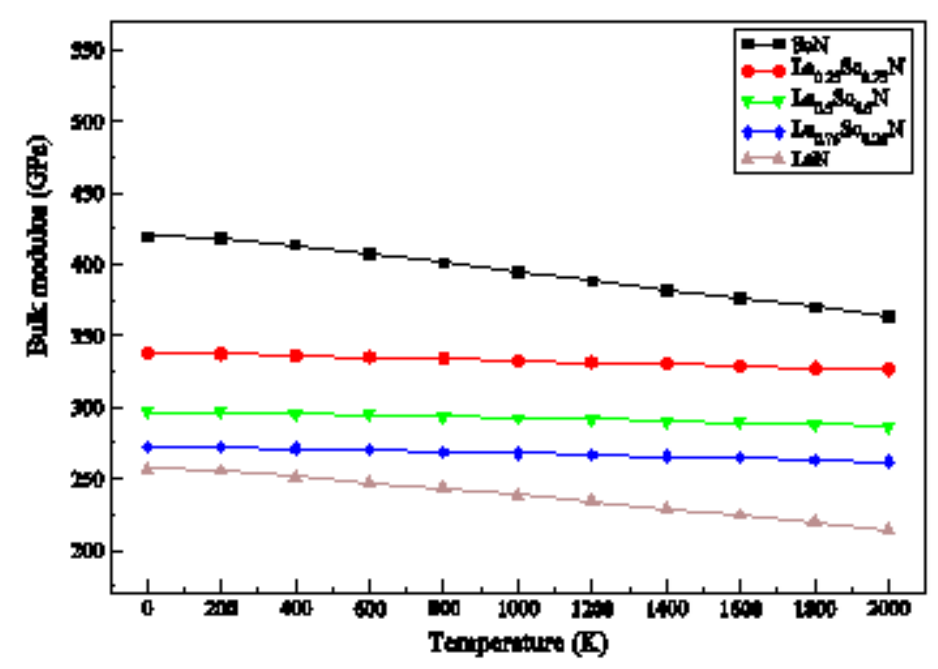

Figure 7

Variation with temperature of the bulk modulus for LaxSc1-xN alloys. 


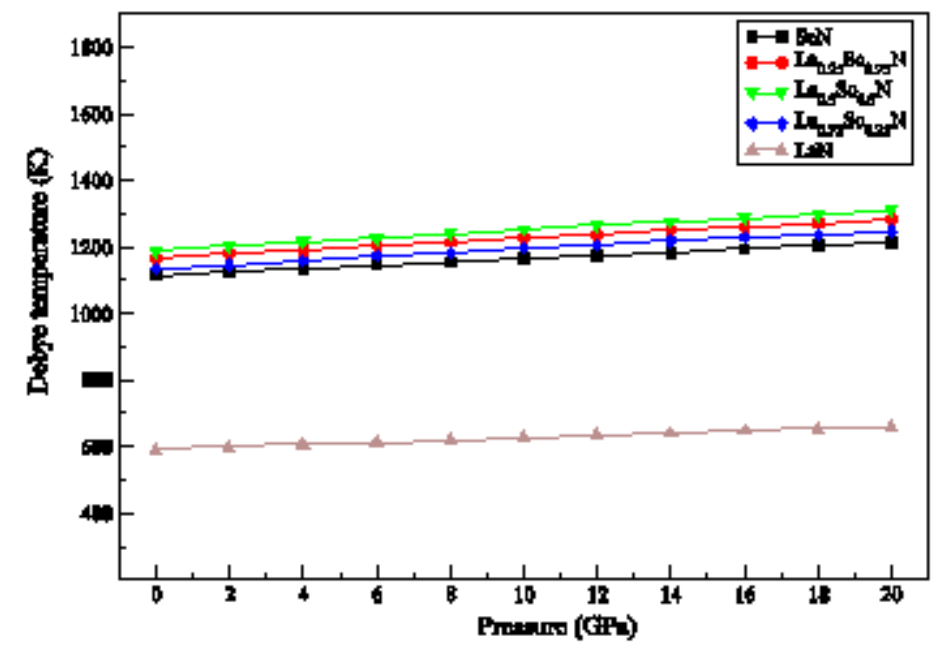

Figure 8

Variation with temperature of the Debye temperature for LaxSc1-xN alloys.

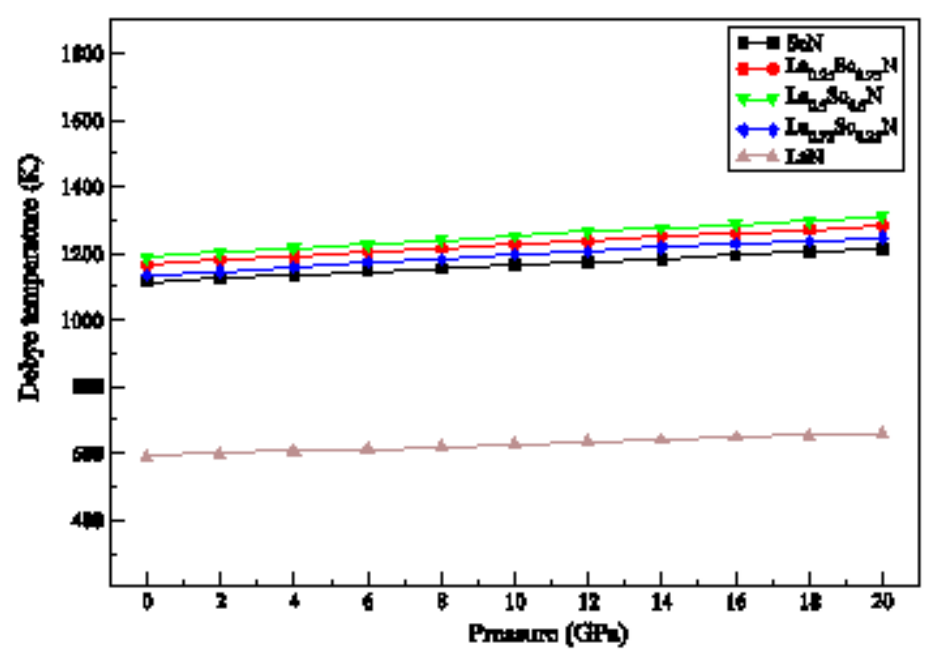

Figure 9

Variation with pressure of the Debye temperature for LaxSc1-xN alloys. 


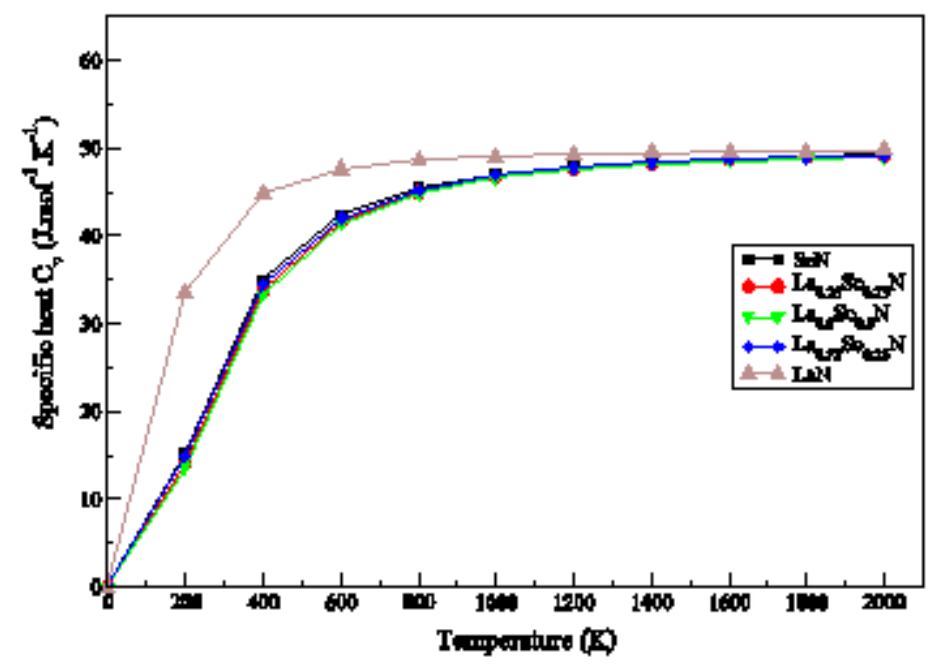

Figure 10

Variation with temperature of the heat capacity (CV) for LaxSc1-xN alloys.

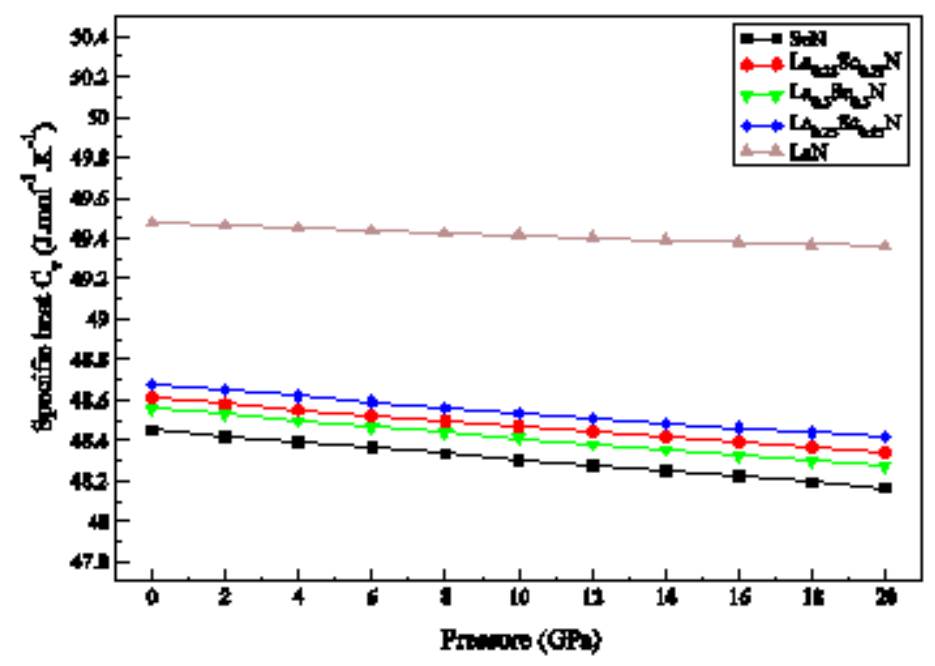

Figure 11

Variation with pressure of the heat capacity (CV) for LaxSc1-xN alloys. 


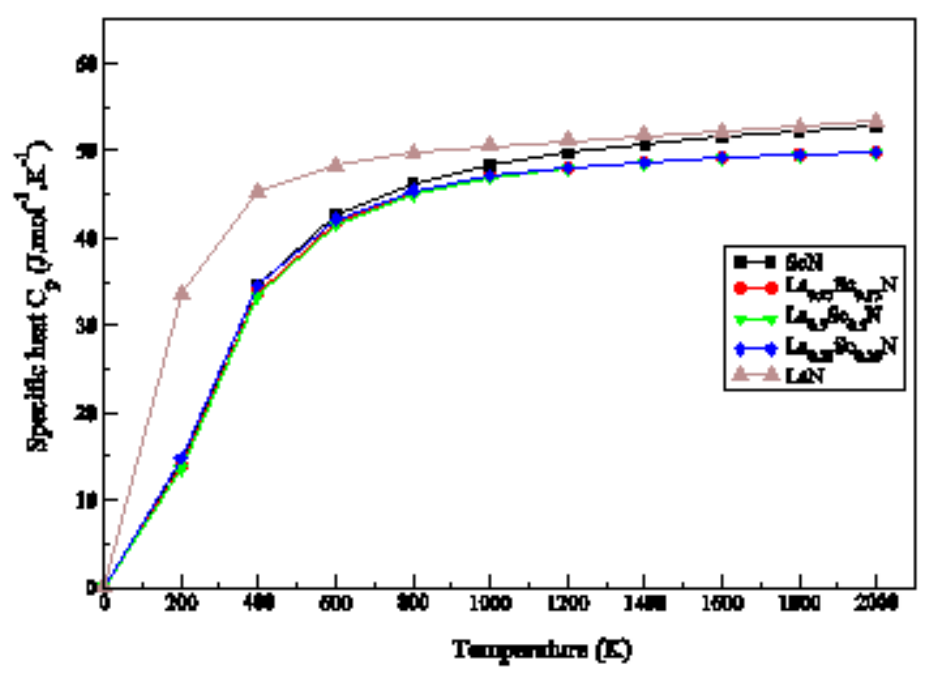

Figure 12

Variation with temperature of the heat capacity (Cp) for LaxSc1-xN alloys

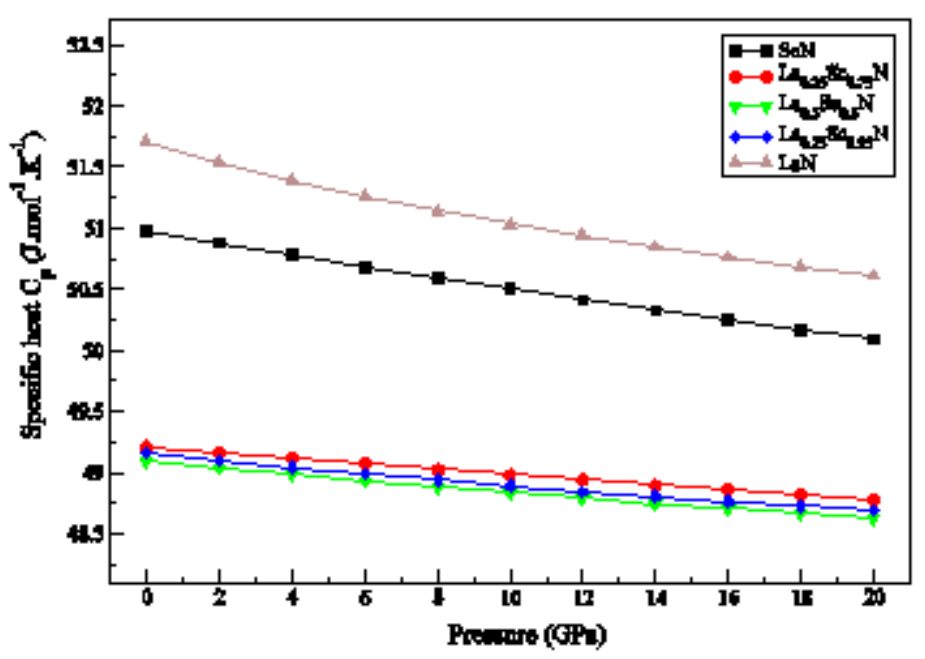

Figure 13

Variation with pressure of the heat capacity (Cp) for LaxSc1-xN alloys. 


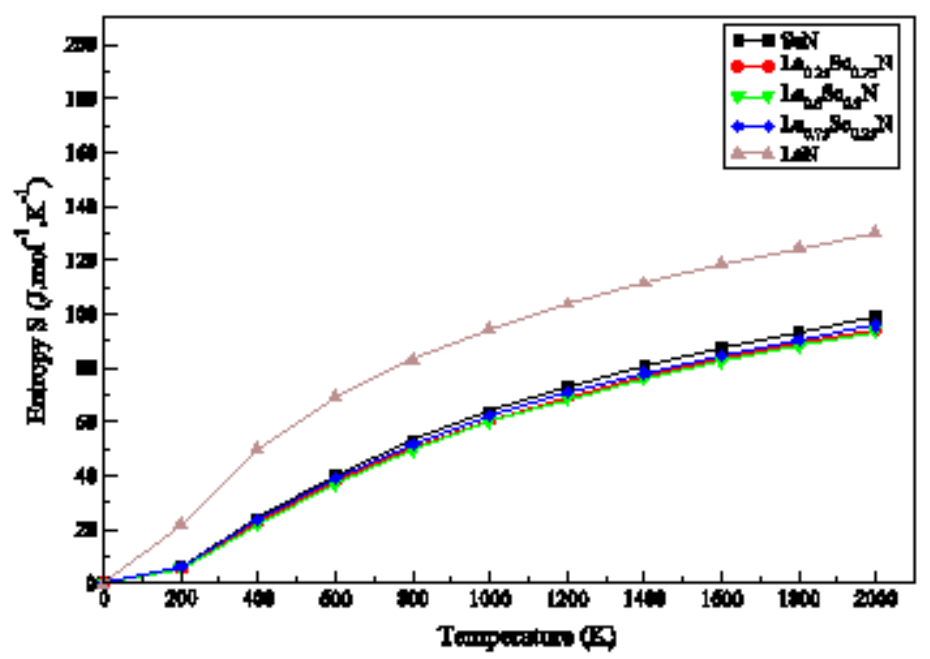

Figure 14

Variation with temperature of the entropy (S) for LaxSc1-xN alloys. 